\title{
EVALUACIÓN DE LA LEGIBILIDAD DE WEBS DE UNIVERSIDADES
}

\author{
José-Ramón Hilera-González, Luis Fernández-Sanz y Esther Suárez-Morón
}
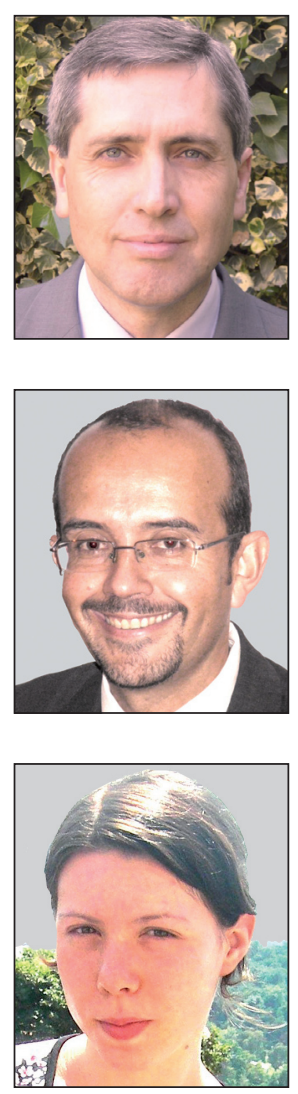

José-Ramón Hilera-González, profesor titular en la Universidad de Alcalá de Henares, imparte docencia en la Facultad de Documentación y en la Escuela de Ingeniería Informática de dicha universidad. Es director del Máster universitario en ingeniería del software para la web y coordinador del programa de doctorado en ingeniería de la información y del conocimiento. Miembro vocal de grupos de trabajo de Aenor, colabora en la elaboración y adaptación de normas UNE de formación virtual y accesibilidad.

Univ. de Alcalá, Dpto. de Ciencias de la Computación, Edif. Politécnico Ctra. Madrid-Barcelona, Km. 33,600. 28871 Alcalá de Henares (Madrid) jose.hilera@uah.es

Luis Fernández-Sanz es licenciado y doctor en informática y profesor titular del Departamento de Ciencias de la Computación en la Universidad de Alcalá de Henares. Vicepresidente de ATI, asociación de profesionales de informática, y editor de la revista Reicis. Sus líneas de investigación incluyen los factores humanos en la ingeniería y la calidad del software así como los modelos de evaluación de calidad de software, incluidos los relacionados con la usabilidad y accesibilidad.

Univ. de Alcalá, Dpto. de Ciencias de la Computación, Edif. Politécnico Ctra. Madrid-Barcelona, Km. 33,600. 28871 Alcalá de Henares (Madrid) http://www.ati.es/reicis luis.fernandezs@uah.es

Esther Suárez-Morón es ingeniera en informática y colabora con el grupo de investigación en tecnologías para la información y el conocimiento (Tifyc) de la Universidad de Alcalá de Henares. Ha trabajado en la empresa privada creando aplicaciones web accesibles y ha participado en la elaboración de un informe sobre la accesibilidad de las páginas web de universidades españolas y extranjeras.

Sopra Group Univ. de Alcalá, Dpto. de Ciencias de la Computación, Edif. Politécnico Ctra. Madrid-Barcelona, Km. 33,600. 28871 Alcalá de Henares (Madrid) esther_su6@hotmail.com

\section{Resumen}

Para conseguir un acceso universal a productos y servicios en la sociedad de la información, que pretende conseguir una Red para todos, hay que garantizar la legibilidad de las páginas web. Se describe un trabajo llevado a cabo para evaluar la legibilidad de contenidos textuales de los portales web de las 20 universidades más importantes del mundo y de las 20 más importantes de España, según rankings internacionales, con el objetivo de comprobar si tienen páginas legibles. Se han aplicado diferentes fórmulas matemáticas que evalúan la dificultad sintáctica de un texto, en los idiomas inglés y español.

\section{Palabras clave}

Legibilidad, Webs de universidades, Accesibilidad web, Escritura digital, Contenido web, Usabilidad.

\section{Title: Evaluation of the readability of university web pages}

\begin{abstract}
Readability refers to the ease of reading and understanding a text. The need to ensure the readability of Web pages is related to universal access to products and services in the information society, which aims to provide an Internet for all. This article describes a study conducted to assess the readability of textual contents of Web portals of 40 universities at the top positions in international university rankings to determine whether the best universities have readable web pages. Mathematical formulas that evaluate syntactic difficulty of text oriented to texts written in English or in Spanish were applied.
\end{abstract}

\section{Keywords}

Readability, Web pages of universities, Web accessibility, Digital writing, Web content, Usability. 


\section{Introducción}

La legibilidad se refiere a la facilidad de lectura y comprensión de un texto. Si un texto es legible, el contenido de un sitio es más fácil de leer para todos y en especial para las personas con discapacidades para la lectura y/o cognitivas (Luján, 2008). Existe una diferencia importante entre legibilidad y comprensión (Sánchez, 2010). Mientras que la primera se refiere a la facilidad con la que las personas lectoras pueden decodificar la información en un documento, la última se refiere al contenido del mismo. Las dos tienen un efecto importante en el éxito o fracaso de la lectura del documento: la comprensión no puede ser adquirida sin la legibilidad (tipoGráfica, 2001).

Trabajos recientes han determinado que los usuarios no leen las páginas completas, sino que escanean la página rápidamente para determinar si merece la pena perder tiempo examinando ese contenido (García-Ferrari; Short, 2002; Nielsen, 2010). La mayoría de los visitantes de una web sólo leen entre un 20 y un $28 \%$ del texto de la página y un $17 \%$ están menos de 4 segundos antes de pasar a otra página. Esto nos da una idea bastante clara del carácter inquieto del internauta (Gil, 2008).

El principal motivo por el que los usuarios únicamente "escanean" es porque leer de la pantalla de un ordenador es cansado para los ojos y un $25 \%$ más lento que la lectura en papel (Rodríguez-López, 2011). Los usuarios tienden a minimizar el número de palabras que leen. Además la web es un medio interactivo, y los usuarios por lo general sienten que tienen que "moverse" todo el tiempo y cliquear. A menudo piensan que sentarse frente a la pantalla y leer no es "productivo". Cada página tiene que competir con cientos de millones de otras para capturar la atención de los navegantes (tipoGráfica, 2001).

La verificación de la legibilidad ha sido considerada desde la creación de la Web un requisito fundamental para garantizar la accesibilidad (Martínez; Hilera-González; FernándezSanz, 2010), y como tal ha sido incluido por el World Wide Web Consortium (W3C) en sus recomendaciones WCAG para conseguir una Web con contenidos accesibles para todo tipo de usuarios (con o sin discapacidad) (W3C, 2008; Voces-Merayo, 2010). En algunos países la necesidad de garantizar la legibilidad de las páginas web está contemplada en la legislación sobre el acceso universal a productos y servicios de la sociedad de la información; y organizaciones de todo el mundo están trabajando en la mejora de la accesibilidad web con el objetivo de conseguir una internet para todos (Vega-Fuente, 2004). Desde este punto de vista, se considera relevante que las webs de las universidades ofrezcan en sus portales textos con un adecuado nivel de accesibilidad y por tanto de legibilidad.

El propósito de este artículo es presentar una evaluación de la legibilidad de las webs de las 20 universidades más importantes del mundo y de las 20 más importantes de España, según rankings internacionales. En primer lugar se describen las técnicas y aplicaciones utilizadas para medir la legibilidad, a continuación se presentan los resultados obtenidos al aplicarlas a dichas webs, y finalmente se discuten los resultados exponiendo las principales conclusiones que pueden derivarse del trabajo realizado.

\section{Material y métodos}

Para medir la dificultad de un texto se utilizan fórmulas matemáticas que evalúan su dificultad sintáctica. Las primeras fórmulas de legibilidad se crearon para la lengua inglesa y se usan extensamente en el campo de la salud. La mayoría de ellas parte de la hipótesis de que cuanto más cortas sean las palabras y frases de un texto más fácil será de leer. No constituyen un método de evaluación exacto, pero sí son indicativas y pueden ser utilizadas junto a otras técnicas complementarias que contemplan aspectos de comprensibilidad semántica y legibilidad tipográfica (Gröne, 2009).

\subsection{Fórmulas empleadas}

Diversos investigadores han propuesto fórmulas para medir el índice o nivel de legibilidad de los textos en diferentes idiomas, especialmente en inglés (DuBay, 2007). De todas las existentes, las que se han usado en este proyecto se muestran en la tabla 1.

\section{RES expresa el grado de comprensibi-} lidad de un texto en inglés en función del promedio de palabras por frase y del promedio de sílabas por palabra

\section{RES (Flesch reading ease score) para idioma inglés}

Este índice expresa el grado de comprensibilidad de un texto en inglés en función del promedio de palabras por frase y del promedio de sílabas por palabra (Flesch, 1948). La fórmula otorga al texto evaluado un valor dentro de la escala de puntos. Cuanto más elevado sea el resultado más fácil será comprender el documento. Para la mayoría de los documentos estándar, el objetivo es un resultado comprendido entre 60 y 70 aproximadamente. EI U. S. Department of Defense utiliza este índice como prueba estándar de legibilidad de sus documentos y formularios; y el estado de Florida requiere que las pólizas de los seguros de vida tengan una puntuación de facilidad de lectura de Flesch de 45 o mayor. Este índice se ha incluido tradicionalmente en la mayoría de programas de procesamiento de texto, entre ellos Microsoft Word ${ }^{2}$.

\section{LECT (Índice de Fernández-Huerta) para idioma español}

Para evaluar documentos en español es necesario adaptar la fórmula de Flesch, debido a que se utilizan frases y pa- 


\begin{tabular}{|l|c|c|}
\hline \multicolumn{1}{|c|}{ Índice } & Fórmula & Autor \\
\hline RES (inglés) & $206,835-1,015 \cdot\left(\frac{\mathrm{n}^{\mathrm{o}} \text { total palabras }}{\mathrm{n}^{\mathrm{o}} \text { total frases }}\right)-84,6 \cdot\left(\frac{\mathrm{n}^{\text {o }} \text { total sílabas }}{\mathrm{n}^{\text {o }} \text { total palabras }}\right)$ & Flesch, 1948 \\
\hline LECT (español) & $L E C T=206,84-0,60 \cdot \mathrm{P}-1,02 \cdot \mathrm{F}$ & Fernández- Huerta, 1959 \\
\hline IFSZ (español) & $206,835-62,3 \cdot \frac{\mathrm{n}^{\circ} \text { total sílabas }}{\mathrm{n}^{\circ} \text { total palabras }}-\frac{\mathrm{n}^{\circ} \text { total palabras }}{\mathrm{n}^{\text {o }} \text { total frases }}$ & Szigriszt, 1993 \\
\hline
\end{tabular}

Tabla 1. Fórmulas de legibilidad

labras más largas que en inglés. A lo largo de los años, la fórmula original de Flesch ha sido adaptada para conseguir una variante válida para español. Algunas de las adaptaciones más importantes se han llevado a cabo en el campo de la didáctica (Fernández-Huerta, 1959), del periodismo (Szigriszt, 1993) y de la salud (Barrio-Cantalejo, 2007). Fernández-Huerta propuso una adaptación de la fórmula RES, utilizando los mismos factores pero con unas ponderaciones diferentes. El resultado fue la expresión de la tabla 1, que determinaba la "lecturabilidad" (LECT) de un texto. Siendo $\mathrm{P}$ el promedio de número de sílabas por cada 100 palabras, y $\mathrm{F}$ el promedio del conjunto de frases por cada 100 palabras.

\section{IFSZ (Índice Flesch-Szigriszt), para idioma español}

La adaptación de la fórmula RES realizada por Szigriszt (1993) se denomina Fórmula de perspicuidad, y es la que se considera de referencia en el momento actual para la lengua española. Además de la anterior, Szigriszt revisó la relación entre el resultado obtenido en la fórmula y el nivel de dificultad de lectura del texto, que Flesch había establecido en siete niveles, desde "muy fácil" hasta "muy difícil" (tabla 2). Para ello partió de la idea de que en los últimos años el nivel cultural de la población se ha elevado considerablemente y, por lo tanto, la percepción de que algo es "fácil" o "difícil" de leer ya no puede ser igual que en tiempos de Flesch (publicó en 1948) (Gröne, 2009). Como resultado, Szigriszt mantuvo los 7 niveles originales del índice RES pero cambió sus puntuaciones (tabla 2).

\begin{tabular}{|l|c|c|}
\hline Nivel de dificultad & $\begin{array}{c}\text { Puntuación } \\
\text { Flesch, 1948 } \\
\text { Fernández-Huerta, 1959 }\end{array}$ & $\begin{array}{c}\text { Puntuación } \\
\text { Szigriszt, 1993 }\end{array}$ \\
\hline Muy fácil & $90-100$ & $85-100$ \\
\hline Fácil & $80-90$ & $75-85$ \\
\hline Bastante fácil & $70-80$ & $65-75$ \\
\hline Normal (para adulto) & $60-70$ & $50-65$ \\
\hline Bastante difícil & $50-60$ & $35-50$ \\
\hline Difícil & $30-50$ & $15-35$ \\
\hline Muy difícil & $0-30$ & $0-15$ \\
\hline
\end{tabular}

Tabla 2. Tabla comparativa entre las diferentes propuestas

\subsection{Software de evaluación automática}

Existen diferentes aplicaciones que pueden medir la legibilidad de un texto y de sitios web evaluando diferentes fórmulas. El programa más empleado es Estadísticas de legibilidad del procesador de textos Microsoft Word, aunque aplica la fórmula RES de Flesch original, y no la versión adaptada por
Szigriszt (Barrio-Cantalejo, 2007). En el trabajo que se presenta en este artículo se han utilizado dos programas para la medición de la legibilidad: Flesh, para idioma inglés; e Inflesz para español.

- Flesh es una aplicación de código abierto realizada en Java, diseñada para examinar rápidamente un documento y mostrar las dificultades asociadas a la comprensión del mismo. Después de procesar un documento ofrece como resultado entre otros indicadores el índice de facilidad de lectura RES de Flesch.

http://flesh.sourceforge.net

- Inflesz, escrito en $\mathrm{C}++$, puede procesar tanto archivos completos como fragmentos de texto y obtiene, entre otros resultados, el valor de la fórmula IFSZ o Índice de Flesch-Szigriszt, y la fórmula LECT o Índice de lecturabilidad de Fernández Huerta.

http://www.legibilidad.com/home/descargas.htm/

\subsection{Selección de la muestra}

Se ha evaluado la legibilidad de los contenidos textuales de las webs de aquellas universidades que aparecen en las primeras posiciones de los rankings universitarios internacionales, con el fin de comprobar si las mejores tienen una web legible, con un texto claro y fácil de entender para la mayoría de los usuarios. Se han revisado tres conocidos rankings mundiales:

- Academic Ranking of World Universities (ARWU) de la Universidad Jiao Tong de Shanghai http://www.arwu.org

- Times Higher Education Supplement (THES) de TSL Education del Reino Unido

http://www.timeshighereducation.co.uk

- Webometrics Rankings of World Universities del Laboratorio de Cibermetría del CSIC de España http://www.webometrics.info

De ellos se ha decidido utilizar la clasificación realizada por ARWU en 2010 para el caso de las webs con texto en inglés, debido a su prestigio y consistencia. Times Higher Education ha sido descartado porque ha ido variando a lo largo de los años su metodología y muestra algunas incoherencias de unas ediciones a otras. Las 20 universidades del mundo elegidas para el análisis pueden verse en la tabla 3.

También se han considerado 20 universidades españolas, tomadas del ranking Webometrics de enero de 2010. Se ha elegido porque fue elaborado el mismo año que la última edición publicada de $A R W U$. Se descartaron los otros rankings porque sólo hay 10 españolas en $A R W U$, y 8 en Times Higher Education (THE 2011-2012). Las presentes en ARWU 


\begin{tabular}{|c|c|c|c|c|c|}
\hline \multicolumn{4}{|c|}{ Acader } & 国 & is availa \\
\hline \multicolumn{2}{|c|}{ 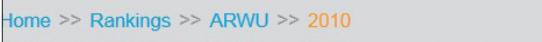 } & \multicolumn{2}{|c|}{ Methodology } & Statistics & Analysis \\
\hline \multicolumn{6}{|c|}{ Academic Ranking of World Universities - 2010} \\
\hline $\begin{array}{l}\text { World } \\
\text { Rank }\end{array}$ & Institution & Country & $\begin{array}{l}\text { National } \\
\text { Rank }\end{array}$ & $\begin{array}{l}\text { Total } \\
\text { Score }\end{array}$ & $\begin{array}{l}\text { Score on } \\
\text { Alumni - }\end{array}$ \\
\hline 1 & Harvard University & 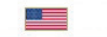 & 1 & 100.0 & 100.0 \\
\hline 2 & University of California, Berkeley & 䨐 & 2 & 72.4 & 67.6 \\
\hline 3 & Stanford University & 暳 & 3 & 72.1 & 40.2 \\
\hline 4 & Massachusetts Institute of Technology (MIT) & 邫 & 4 & 71.4 & 70.5 \\
\hline 5 & University of Cambridge & 果 & 1 & 69.6 & 88.5 \\
\hline 6 & California Institute of Technology & 㕩 & 5 & 64.4 & 50.3 \\
\hline 7 & Princeton University & 㹃 & 6 & 60.8 & 56.4 \\
\hline 8 & Columbia University & 墨 & 7 & 60.4 & 70.7 \\
\hline 9 & University of Chicago & 吿 & 8 & 57.3 & 65.5 \\
\hline 10 & University of Oxford & 为 & 2 & 56.4 & 56.2 \\
\hline
\end{tabular}

Figura 1. Primeras posiciones del ranking ARWU de 2010

2010 y THE 2011-2012 también están incluidas en el ranking utilizado, pero en diferentes posiciones.

http://www.webometrics.info/premierleague_es.htm/

Las universidades españolas analizadas pueden verse en la tabla 4 (se indica si pertenecen también a los otros rankings).

Se ha realizado el análisis de una página web perteneciente a cada universidad. En general se ha seleccionado la relacionada con la historia del centro debido a que suele ser la página menos técnica y más literaria del portal, su tamaño es extenso y permite disponer de suficiente texto para analizar la legibilidad. Sólo en algunos casos, debido al escaso texto de ésta, se ha buscado una página alternativa. En las tablas 3 y 4 se indica el URL de la página examinada en cada caso.

\section{Resultados}

Para analizar las páginas en inglés, como se indicó en el apartado 2, se ha aplicado la fórmula RES (Flesch Reading Ease Score) utilizando la aplicación informática Flesh. Los resultados se muestran en la tabla 5 .

La mitad de las universidades analizadas se localizan en el rango "Difícil", por encontrarse entre los valores 30 y 50. Ha obtenido la mejor puntuación dentro de esta categoría la University of Washington (43 puntos). Por el contrario la University of Pennsylvania ha obtenido la puntuación más baja (31), situándose muy cerca del rango denominado "Muy difícil". Las otras diez se localizan en el rango "Muy difícil", ya que se encuentran entre los valores 0 y 30. La University of California en Los Ángeles ha obtenido el valor más bajo de legibilidad (12 puntos).

Para realizar el análisis de las páginas de las universidades cuyo idioma principal es el español, se ha utilizado el programa Inflesz. A diferencia de Flesh utiliza dos fórmulas: fórmula de Szigriszt y fórmula de Fernández-Huerta, descritas en el apartado 2, obteniendo así unos resultados más pre-

\begin{tabular}{|c|c|}
\hline Universidad & URL de la página examinada \\
\hline Harvard Univ. & http://www.news.harvard.edu/guide/content/history-harvard-university \\
\hline Univ. of California, Berkeley & http://berkeley.edu/about/hist \\
\hline Stanford Univ. & http://www.stanford.edu/about/history \\
\hline Massachusetts Institute of Technology & http://info-libraries.mit.edu/mithistory/institute/seal-of-the-massachusetts-institute-of-technology \\
\hline Univ. of Cambridge & http://www.cam.ac.uk/univ/history/post1945.html \\
\hline California Institute of Techonology & http://admissions.caltech.edu/about/history \\
\hline Princeton Univ. & http://www.princeton.edu/main/about/history/glance \\
\hline Columbia Univ. & http://www.columbia.edu/content/history.html \\
\hline Univ. of Chicago & http://www.uchicago.edu/about/history.shtml \\
\hline Univ. of Oxford & http://www.ox.ac.uk/about_the_university/introducing_oxford/a_brief_history_of_the_university/index.html \\
\hline Yale Univ. & http://www.yale.edu/about/history.html \\
\hline Cornell Univ. & http://landgrant.cornell.edu/history.cfm \\
\hline Univ. of California, Los Angeles & http://www.ucla.edu/about.html \\
\hline Univ. of California, San Diego & http://www.ucsd.edu/explore/about/index.html \\
\hline Univ. of Pennsylvania & http://www.upenn.edu/about/heritage.php \\
\hline Univ. of Washington & http://www.washington.edu/discover \\
\hline Univ. of Wisconsin, Madison & http://www.wisc.edu/about/history \\
\hline The Johns Hopkins Univ. & http://webapps.jhu.edu/jhuniverse/information_about_hopkins/about_jhu/a_brief_history_of_jhu/index.cfm \\
\hline Univ. of California, San Francisco & http://www.ucsf.edu/about/history-1 \\
\hline Univ. of Tokyo & http://www.u-tokyo.ac.jp/en/about/president.html \\
\hline
\end{tabular}

Tabla 3. Webs examinadas en inglés 


\begin{tabular}{|c|c|}
\hline Universidad & URL examinada \\
\hline Univ. Complutense de Madrid (ARWU) & http://www.ucm.es/pags.php?tp=Historia de la Universidad Complutense $\& a=\& d=0000624 . p h p$ \\
\hline Univ. Politécnica de Madrid & http://www.upm.es/institucional/UPM/Historia/ResenaHistorica \\
\hline Univ. de Sevilla & http://www.us.es/acerca/bienvenida/index.html \\
\hline Univ. de Barcelona (ARWU, THE) & http://www.ub.edu/web/ub/es/universitat/coneix_la_ub/visites_a_la_universitat/visites.html \\
\hline Univ. Politècnica de Catalunya (THE) & http://www.upc.edu/upc/la-institucion/el-rector \\
\hline Univ. Autònoma de Barcelona (ARWU, THE) & http://www.uab.es/servlet/Satellite/informacion-academica-de-grados-1289285283209.html \\
\hline Univ. de Granada (ARWU) & http://www.ugr.es/pages/universidad/granada \\
\hline Univ. d'Alacant & http://web.ua.es/es/universidad-alicante/antecedentes-y-evolucion.html \\
\hline Univ. del País Vasco & http://www.ehu.es/p200-content/es/contenidos/informacion/historia_upvehu/es_histori1/historia_1.html \\
\hline Univ. Politécnica de Valencia (ARWU, THE) & http://www.upv.es/organizacion/conoce-upv/historia/historia1-es.html \\
\hline Univ. de Murcia & http://www.um.es/universidad/historia-umu/precedentes.php \\
\hline Univ. de València (ARWU, THE) & http://www.uv.es/ webuv/castellano/informacio/presentacio_historia.htm \\
\hline Univ. de Zaragoza (ARWU, THE) & http://www.unizar.es/resena_historica.html \\
\hline Univ. de Salamanca & http://www.usal.es/webusal/node/941 \\
\hline Univ. Autónoma de Madrid (ARWU, THE) & $\begin{array}{l}\text { http://www.uam.es/ss/Satellite/es/1233310432203/contenidoFinal/10_razones_para_estudiar_en_la_ } \\
\text { Universidad_Autonoma_de_Madrid.htm?idenlace }=1233310440300\end{array}$ \\
\hline Univ. de Vigo & http://uvigo.es/uvigo_es/organizacion/informacion/index.html \\
\hline Univ. Nacional de Educación a Distancia & http://portal.uned.es/portal/page?_pageid=93,499271,93_20500119\&_dad=portal\&_schema=PORTAL \\
\hline Univ. de Santiago de Compostela (ARWU) & http://www.usc.es/es/info_xeral/historia \\
\hline Univ. de les Illes Baleares & http://www.uib.es/es/lauib \\
\hline Univ. Pompeu Fabra (ARWU, THE) & http://www.upf.edu/universitat/es/presentacio/historia.html \\
\hline
\end{tabular}

Tabla 4. Webs examinadas en español

\begin{tabular}{|c|c|c|c|}
\hline Portal & $\begin{array}{l}\text { Posición } \\
\text { en ranking } \\
\text { ARWU }\end{array}$ & $\begin{array}{c}\text { Índice de } \\
\text { legibilidad } \\
\text { RES }\end{array}$ & $\begin{array}{l}\text { Nivel de } \\
\text { dificultad }\end{array}$ \\
\hline 1. Univ. of Washington & 16 & 43 & Difícil \\
\hline 2. Harvard Univ. & 1 & 42 & Difícil \\
\hline 3. Stanford Univ. & 3 & 39 & Difícil \\
\hline 4. Univ. of Oxford & 10 & 36 & Difícil \\
\hline $\begin{array}{l}\text { 5. California Institute } \\
\text { of Techonology }\end{array}$ & 6 & 34 & Difícil \\
\hline 6. Yale Univ. & 11 & 34 & Difícil \\
\hline $\begin{array}{l}\text { 7. Univ. of California, } \\
\text { San Francisco }\end{array}$ & 19 & 33 & Difícil \\
\hline 8. Princeton Univ. & 7 & 32 & Difícil \\
\hline 9. Columbia Univ. & 8 & 31 & Difícil \\
\hline $\begin{array}{l}\text { 10. Univ. of } \\
\text { Pennsylvania }\end{array}$ & 15 & 31 & Difícil \\
\hline $\begin{array}{l}\text { 11. Univ. of California, } \\
\text { Berkeley }\end{array}$ & 2 & 28 & Muy difícil \\
\hline 12. Univ. of Tokyo & 20 & 27 & Muy difícil \\
\hline 13. Univ. of Chicago & 9 & 24 & Muy difícil \\
\hline $\begin{array}{l}\text { 14. Univ. of Wisconsin } \\
\text { - Madison }\end{array}$ & 17 & 22 & Muy difícil \\
\hline $\begin{array}{l}\text { 15. The Johns Hopkins } \\
\text { Univ. }\end{array}$ & 18 & 20 & Muy difícil \\
\hline 16. Univ. of Cambridge & 5 & 19 & Muy difícil \\
\hline 17. Cornell Univ. & 12 & 18 & Muy difícil \\
\hline $\begin{array}{l}\text { 18. Massachusetts } \\
\text { Institute of } \\
\text { Technology }\end{array}$ & 4 & 18 & Muy difícil \\
\hline $\begin{array}{l}\text { 19. Univ. of California, } \\
\text { San Diego }\end{array}$ & 14 & 16 & Muy difícil \\
\hline $\begin{array}{l}\text { 20. Univ. of California, } \\
\text { Los Angeles }\end{array}$ & 13 & 12 & Muy difícil \\
\hline
\end{tabular}

Tabla 5. Análisis de la legibilidad en páginas con idioma inglés aplicando la fórmula Flesh Reading Ease Level cisos y contrastados. Los resultados obtenidos al aplicar la primera de ellas se muestra en la tabla 6.

Las universidades Politécnica de Madrid, UNED y de Barcelona son las que han obtenido los mejores resultados, con una puntuación igual o superior a 60, localizándose dentro del rango "Normal" en la clasificación de Szigriszt. La que ha obtenido la mejor puntuación dentro de la categoría "Normal" ha sido la Universitat de Barcelona, con una puntuación de 63; por el contrario, la Universidad de Salamanca ha obtenido la puntuación más baja en esta categoría (50). La Politècnica de Valencia, Complutense de Madrid, Sevilla y Autónoma de Barcelona se encuentran entre los rangos "Normal" y "Bastante difícil", ya que sus valores son muy cercanos a 50 . Las universidades de Granada, Santiago de Compostela, Autónoma de Madrid, València y Murcia se localizan claramente en el rango de "Bastante difícil". En este rango se encuentran también las de Alicante y Vigo, pero muy cerca de la categoría "Difícil", en la que por pocas décimas está incluida la Universidad de las Islas Baleares. La universidad con peor índice de legibilidad ha sido la Politècnica de Catalunya, la única en la categoría "Muy difícil".

Se ha repetido el análisis con el mismo programa, pero aplicando la fórmula de Fernández-Huerta. En este caso los resultados son los indicados en la tabla 7.

En este segundo análisis, el orden de clasificación ha sido prácticamente el mismo que en el caso anterior, excepto para las del País Vasco y Politècnica de València, que por pocas décimas intercambian su posición. En cuanto al nivel de dificultad, con el índice de Fernández-Huerta 4 centros que con la fórmula de Szigriszt tenían un nivel "Normal", pasan a tener un nivel "Bastante difícil", y 7 de nivel "Bastante difícil", tendrían ahora un nivel "Difícil". 


\begin{tabular}{|c|c|c|c|}
\hline Web & $\begin{array}{l}\text { Posición } \\
\text { en ranking } \\
\text { Webometrics }\end{array}$ & $\begin{array}{l}\text { Índice de } \\
\text { legibilidad } \\
\text { IFSZ }\end{array}$ & $\begin{array}{l}\text { Nivel de } \\
\text { dificultad }\end{array}$ \\
\hline $\begin{array}{l}\text { 1. Univ. Politécnica de } \\
\text { Madrid }\end{array}$ & 2 & 63 & Normal \\
\hline $\begin{array}{l}\text { 2. Univ. Nacional } \\
\text { de Educación a } \\
\text { Distancia }\end{array}$ & 17 & 63 & Normal \\
\hline 3. Univ. de Barcelona & 4 & 60 & Normal \\
\hline 4. Univ. Pompeu Fabra & 20 & 59 & Normal \\
\hline 5. Univ. de Zaragoza & 13 & 55 & Normal \\
\hline 6. Univ. del País Vasco & 9 & 50 & Normal \\
\hline $\begin{array}{l}\text { 7. Univ. Politècnica de } \\
\text { València }\end{array}$ & 10 & 50 & Normal \\
\hline 8. Univ. de Salamanca & 14 & 50 & Normal \\
\hline $\begin{array}{l}\text { 9. Univ. Complutense } \\
\text { de Madrid }\end{array}$ & 1 & 49 & $\begin{array}{l}\text { Bastante } \\
\text { difícil }\end{array}$ \\
\hline 10. Univ. de Sevilla & 3 & 49 & $\begin{array}{l}\text { Bastante } \\
\text { difícil }\end{array}$ \\
\hline $\begin{array}{l}\text { 11. Univ. Autónoma de } \\
\text { Barcelona }\end{array}$ & 6 & 49 & $\begin{array}{l}\text { Bastante } \\
\text { difícil }\end{array}$ \\
\hline 12. Univ. de Granada & 7 & 43 & $\begin{array}{l}\text { Bastante } \\
\text { difícil }\end{array}$ \\
\hline $\begin{array}{l}\text { 13. Univ. de Santiago de } \\
\text { Compostela }\end{array}$ & 18 & 42 & $\begin{array}{l}\text { Bastante } \\
\text { difícil }\end{array}$ \\
\hline $\begin{array}{l}\text { 14. Univ. Autónoma de } \\
\text { Madrid }\end{array}$ & 15 & 40 & $\begin{array}{l}\text { Bastante } \\
\text { difícil }\end{array}$ \\
\hline 15. Univ. de València & 12 & 39 & $\begin{array}{l}\text { Bastante } \\
\text { difícil }\end{array}$ \\
\hline 16. Univ. de Murcia & 11 & 38 & $\begin{array}{l}\text { Bastante } \\
\text { difícil }\end{array}$ \\
\hline 17. Univ. d'Alacant & 8 & 36 & $\begin{array}{l}\text { Bastante } \\
\text { difícil }\end{array}$ \\
\hline 18. Univ. de Vigo & 16 & 35 & $\begin{array}{l}\text { Bastante } \\
\text { difícil }\end{array}$ \\
\hline $\begin{array}{l}\text { 19. Univ. de les Illes } \\
\text { Baleares }\end{array}$ & 19 & 35 & Difícil \\
\hline $\begin{array}{l}\text { 20. Univ. Politècnica de } \\
\text { Catalunya }\end{array}$ & 5 & 14 & Muy difícill \\
\hline
\end{tabular}

Tabla 6. Resultados de la legibilidad utilizando la fórmula de Szigriszt

\section{Discusión}

En la evaluación de la legibilidad de las webs de las universidades con idioma inglés se puede destacar que las páginas examinadas han obtenido unos niveles de dificultad muy elevados en comparación con las españolas. De las 20 páginas evaluadas, la mitad presentan textos con dificultad de lectura "Difícil", y la otra mitad un nivel "Muy difícil". La University of California en Los Ángeles ha sido la que ha obtenido el peor resultado. En conclusión se puede ver que todas las universidades de habla inglesa examinadas tienen unos resultados bajos en cuanto a legibilidad.

Si comparamos la posición de las universidades en el ranking $A R W U$ y en el obtenido sobre legibilidad, podemos concluir que no se puede afirmar que exista una relación directa entre ambos, pues sólo 5 de las 10 primeras de ARWU se encuentran entre las 10 de mayor legibilidad. Esta relación sí se cumple en el caso de dos universidades: Harvard y Stanford, ya que estando entre las tres mejor valoradas a nivel mundial, también están entre las tres con mejor le-

\begin{tabular}{|c|c|c|c|}
\hline Web & $\begin{array}{l}\text { Posición } \\
\text { en ranking } \\
\text { Webometrics }\end{array}$ & $\begin{array}{l}\text { Índice de } \\
\text { legibilidad } \\
\text { LECT }\end{array}$ & $\begin{array}{l}\text { Nivel de } \\
\text { dificultad }\end{array}$ \\
\hline $\begin{array}{l}\text { 1. Univ. Politécnica de } \\
\text { Madrid }\end{array}$ & 2 & 68 & Normal \\
\hline $\begin{array}{l}\text { 2. Univ. Nacional } \\
\text { de Educación a } \\
\text { Distancia }\end{array}$ & 17 & 67 & Normal \\
\hline 3. Univ. de Barcelona & 4 & 65 & Normal \\
\hline 4. Univ. Pompeu Fabra & 20 & 64 & Normal \\
\hline 5. Univ. de Zaragoza & 13 & 60 & $\begin{array}{c}\text { Bastante } \\
\text { difícil }\end{array}$ \\
\hline $\begin{array}{l}\text { 6. Univ. Politècnica de } \\
\text { València }\end{array}$ & 10 & 55 & $\begin{array}{c}\text { Bastante } \\
\text { difícil }\end{array}$ \\
\hline 7. Univ. del País Vasco & 9 & 55 & $\begin{array}{c}\text { Bastante } \\
\text { difícil }\end{array}$ \\
\hline 8. Univ. de Salamanca & 14 & 55 & $\begin{array}{l}\text { Bastante } \\
\text { difícil }\end{array}$ \\
\hline $\begin{array}{l}\text { 9. Univ. Complutense } \\
\text { de Madrid }\end{array}$ & 1 & 54 & $\begin{array}{l}\text { Bastante } \\
\text { difícil }\end{array}$ \\
\hline 10. Univ. de Sevilla & 3 & 53 & $\begin{array}{c}\text { Bastante } \\
\text { difícil }\end{array}$ \\
\hline $\begin{array}{l}\text { 11. Univ. Autónoma de } \\
\text { Barcelona }\end{array}$ & 6 & 53 & $\begin{array}{c}\text { Bastante } \\
\text { difícil }\end{array}$ \\
\hline 12. Univ. de Granada & 7 & 48 & Difícil \\
\hline $\begin{array}{l}\text { 13. Univ. de Santiago de } \\
\text { Compostela }\end{array}$ & 18 & 46 & Difícil \\
\hline $\begin{array}{l}\text { 14. Univ. Autónoma de } \\
\text { Madrid }\end{array}$ & 15 & 45 & Difícil \\
\hline 15. Univ. de València & 12 & 43 & Difícil \\
\hline 16. Univ. de Murcia & 11 & 42 & Difícil \\
\hline 17. Univ. d'Alacant & 8 & 42 & Difícil \\
\hline 18. Univ. de Vigo & 16 & 40 & Difícil \\
\hline $\begin{array}{l}\text { 19. Univ. de les Illes } \\
\text { Baleares }\end{array}$ & 19 & 39 & Difícil \\
\hline $\begin{array}{l}\text { 20. Univ. Politécnica de } \\
\text { Catalunya }\end{array}$ & 5 & 19 & Muy difícil \\
\hline
\end{tabular}

Tabla 7. Resultados de la legibilidad utilizando la fórmula de FernándezHuerta

gibilidad. Sin embargo esto no ocurre con la segunda del ranking mundial, la University of California en Berkeley, que está en la posición undécima de legibilidad, con un nivel "Muy difícil".

En cuanto a las universidades con idioma español se puede destacar que de las 20 analizadas, únicamente cuatro presentan textos con una legibilidad "Normal", al ser evaluadas tanto con la fórmula Szigristz como con la de Fernández-Huerta. Son la Politécnica de Madrid, UNED, Barcelona y Pompeu Fabra; y representan un porcentaje del $20 \%$. En el caso de la fórmula de Szigristz, además de las dos anteriores, las de Zaragoza, País Vasco, Politècnica de València y Salamanca también contienen textos de dificultad "Normal", con lo que el porcentaje aumentaría a un $40 \%$. En las páginas del rango "Bastante difícil" también existen diferencias entre ambas fórmulas. En ambos casos coinciden tres universidades, siendo el porcentaje del $50 \%$ según la fórmula de Szigristz y del 35\% según la de Fernández-Huerta. En las páginas con dificultad de lectura más elevada, destaca la Universitat Politècnica de Catalunya.

Al igual que ocurría en el caso de los textos en inglés, tampoco en el caso de las webs con texto en español se puede 
afirmar que exista un relación directa entre el ranking Webometrics y el ranking de legibilidad obtenido. Sólo 5 de las 10 primeras de Webometrics se encuentran entre las 10 de mayor legibilidad en español. Esta relación sólo se cumple en el caso de la Universidad Politécnica de Madrid, que es la segunda mejor valorada en Webometrics, y la que tiene mejor legibilidad.

A partir del trabajo realizado se ha llegado a la conclusión de que en la mayoría de los sitios examinados los textos son difíciles de leer y comprender, especialmente en el caso de las páginas con idioma inglés. Esto provoca que el contenido sea menos accesible para todos los usuarios, pero en especial para las personas con discapacidades para la lectura y/o cognitivas. Aunque es cierto que se trata de páginas universitarias y por tanto dirigidas principalmente a estudiantes y profesores, en lo posible sería conveniente que la información se transmitiera de tal forma que pudiera ser comprendida con facilidad por la mayoría de usuarios que puedan acceder a los portales de las universidades, aunque no se trate de profesores o estudiantes, sino de personas con interés por conocer los detalles y la historia de cada universidad.

\section{Notas}

1. Este trabajo ha sido financiado en parte por la Comisión Europea a través del proyecto DCI-ALA/19.09.01/11/21526/ 279-146/ALFA 111(2011)-11, y por la Comunidad de Madrid y la Universidad de Alcalá a través del proyecto CCG10-UAH/ TIC-5915.

2. En Word 2002 hay que ir al menú "Herramientas", "Opciones", "Ortografía y gramática", y marcar la casilla "Legibilidad". Luego de abrir el archivo que se desee, y después de revisar la ortografía y la gramática aparecen los datos y los promedios: caracteres por palabra, palabras por oración y oraciones por párrafo. Es incompleto porque no calcula el número de sílabas por palabra. En cambio Word 2007 sí calcula el promedio de sílabas por palabra, y en esta versión del programa hay que ir al menú "Archivo".

\section{Bibliografía}

Barrio-Cantalejo, Inés. Legibilidad y salud. Los métodos de medición de la legibilidad y su aplicación al diseño de folletos educativos sobre salud. Tesis doctoral. Universidad Autónoma de Madrid, 2007.

http://tdx.cat/handle/10803/49718

DuBay, William. Smart language: readers, readability, and the grading of text. Costa Mesa (Estados Unidos): Impact Information, 2007. ISBN: $141965439 \mathrm{X}$ http://www.nald.ca/library/learning/smartlang/smartlang. $p d f$

Fernández-Huerta, José. "Medidas sencillas de lecturabilidad". Consigna, 1959, n. 214, pp. 29-32.

Flesch, Rudolph. "A new readability yardstick". Journal of applied psychology, 1948, v. 32, n. 3, pp. 221-233.

http://dx.doi.org/10.1037/h0057532
García-Ferrari, Tomás; Short, Carolina. Legibilidad y comprensión en la world wide web. Bigital, 2002. http://bigital.com/castellano/files/2009/01/legibilidad_ pantalla.pdf

Gil, José-Carlos. “¿Cómo lee la gente en internet?”. Wezstudio, 2008.

http://www.wezstudio.com/blog/como-lee-la-gente-eninternet/

Gröne, Oana. Inventario de instrumentos para medir la legibilidad de un texto en castellano. HPH Catalunya, 2009. http://www.udg.edu/LinkClick.aspx?fileticket=7Uf8HjTWIU\%3D\&tabid $=15493$ \&language $=$ ca-ES

Luján, Sergio. "Legibilidad: facilidad de lectura y comprensión". Accesibilidad en la Web, 14 octubre 2008.

http://accesibilidadenlaweb.blogspot.com/2008/10/legibi lidad-facilidad-de-lectura-y.html

Martínez, Miriam; Hilera-González, José-Ramón; Fernández-Sanz, Luis. "Readability metrics for web applications accessibility". En: $12^{\text {th }}$ Int l conf on enterprise information systems, 2010, pp. 207-210.

Nielsen, Jakob. "Why web users scan instead of read". Useit. com, 2010.

http://www.useit.com/alertbox/whyscanning.html

Rodríguez-López, Joaquín. "La contracción digital del presente". El profesional de la información, 2011, marzo-abril, v. 20, n. 2 , pp. $219-227$.

http://dx.doi.org/10.3145/epi.2011.mar.14

Sánchez, Nuria. "Legibilidad de una página web". Blog de posicionamiento web, 2010.

http://www.iniziar.com/blog/optimizacion-web/legibilidadde-una-pagina-web

Szigriszt, Francisco. Sistemas predictivos de legibilidad del mensaje escrito: fórmula de perspicuidad. Tesis doctoral, Universidad Complutense de Madrid, 1993.

http://eprints.ucm.es/1785

TipoGráfica. "Legibilidad y comprensión en la Web". TipoGráfica, 2001.

http://tpgbuenosaires.tipografica.com/workshops/apun tes/tipografia_en_la_web.html

Vega-Fuente, Amando. "Internet para todos: después del año europeo de la discapacidad". Comunicar: revista científica de comunicación y educación, 2004, n. 22, pp. 141-151. http://rabida.uhu.es/dspace/bitstream/handle/10272/ 1157/b15239214.pdf?sequence $=1$

Voces-Merayo, Ramón. "Diseño de arquitecturas de información lineales para mejorar la accesibilidad web". El profesional de la información, 2010, julio-agosto, v. 19, n. 4, pp. 374-381.

http://dx.doi.org/10.3145/epi.2010.jul.06

W3C. Web content accessibility guidelines (WCAG) 2.0. World Wide Web Consortium, 2008.

http://www.w3.org/TR/WCAG 